\title{
LATS1 suppresses proliferation and invasion of cervical cancer
}

\author{
JIHONG DENG, WEN ZHANG, SHUANGYUE LIU, HONGMEI AN, LU TAN and LISHA MA \\ Department of Gynecology, Kunming Maternity and Child Care Hospital, Kunming, Yunnan 650000, P.R. China
}

Received December 14, 2015; Accepted February 3, 2016

DOI: $10.3892 / \mathrm{mmr} .2017 .6180$

\begin{abstract}
Loss of large tumor suppressor kinase 1 (LATS1)Y has been implicated in numerous types of human cancer. However, its involvement in human cervical cancer remains to be elucidated. The present study aimed to investigate the clinical significance and biological characteristics of LATS1 in human cervical cancer. The present study investigated the protein expression levels of LATS1 in tissues from 80 cases of cervical cancer using immunohistochemistry and demonstrated that LATS1 was downregulated in $45 \%(36 / 80)$ of cervical cancers. Transfection of LATS1 was performed in the SiHa cell line and LATS1 siRNA knockdown was performed in the Caski cell line. MTT assay and Matrigel invasion assay indicated that LATS1 overexpression inhibited cell proliferation and invasion. LATS1 overexpression upregulated p27 expression, and downregulated the expression of cyclin E and matrix metalloproteinase 9. In addition, LATS1 overexpression stimulated yes-associated protein 1 (YAP) phosphorylation. Depletion of LATS1 in Caski cells resulted in the opposite effects. The current study demonstrated that LATS1 was downregulated in cervical cancer and may suppress cell growth and invasion through regulating the expression of cyclin E, p27, MMP9 and YAP.
\end{abstract}

\section{Introduction}

Cervical cancer is the third most common malignancy in women worldwide, with a global incidence of 500,000 and a mortality of 250,000 in 2014 (1). For the earliest stage of cervical cancer, $>90 \%$ of women survive at least 5 years after diagnosis. However, advanced cervical cancer with invasion or metastasis is associated with a poor prognosis, $<20 \%$ of stage IV patients survive for $\geq 5$ years (2). Furthermore, despite advances of conventional therapies such as surgical treatment, radiotherapy and chemotherapy, malignant cervical cancers still have high mortality rate, and the mechanism underlying its aggressiveness remains poorly understood. Thus, the

Correspondence to: Dr Jihong Deng, Department of Gynecology, Kunming Maternity and Child Care Hospital, 5 West Huashan Road, Kunming, Yunnan 650000, P.R. China

E-mail: jihongdeng@126.com

Key words: LATS1, cervical cancer, proliferation, invasion identification of novel molecular markers, which is helpful for the development of novel diagnostic and therapeutic strategies, remains an important focus in the current management of this malignancy.

Mammalian Large tumor suppressor kinase 1 (LATS1) and LATS2, the major kinase components of the Hippo pathway, are important in the control of tumor development $(3,4)$ and the cell cycle, via various mechanisms and signaling pathways $(5,6)$. It was previously reported that LATS1 protein was downregulated in various types of cancer, including breast carcinoma (7), colorectal carcinoma (8), gastric cancer (9), non-small cell lung cancer (10) and ovarian serous carcinoma and clear cell carcinoma (11). These results indicated that LATS1 may be an important tumor suppressor in types of human cancer. However, whether LATS1 is a tumor suppressor in cervical cancer remains controversial.

The present study examined LATS1 protein expression in 80 cases of cervical carcinoma and analyzed the association between LATS1 expression and clinicopathological factors. Additionally, gain of function and loss of function experiments were performed to investigate the biological roles of LATS1 in cervical cancer. LATS1 expression was upregulated in SiHa cells and depleted in Caski cells, and the effects on cell proliferation and invasion were then examined. In addition, the molecular signaling pathways underlying these biological effects of LATS1 were investigated.

\section{Materials and methods}

Patients and specimens. The protocol of the current study was approved by the Institutional Review Board of Kunming Maternity and Child Care Hospital (Kunming, China). Primary tumor specimens were obtained from 80 patients (mean age, 45.5; range, 28-72) diagnosed with cervical carcinoma who underwent resection in Kunming Maternity and Child Care Hospital between January 2012 and November 2014. Informed consent was obtained. Histological diagnosis was performed on sections stained with hematoxylin and eosin, according to the World Health Organization classification guidelines (12). Clinical and histopathological data were obtained from medical records.

Immunohistochemistry. Cervical cancer tissue specimens were fixed in $10 \%$ formalin at room temperature for $24 \mathrm{~h}$ and embedded in paraffin. Immunohistochemistry was carried out using Elivision ${ }^{\mathrm{TM}}$ plus Polyer HRP IHC kit (Fuzhou Maixin Biotech Co., Ltd., Fuzhou, China) according 
to the manufacturer-s protocol. Briefly, $4 \mu \mathrm{m}$ thick tissue sections were deparaffinized and rehydrated using ethanol. Subsequently, antigen retrieval was performed using $0.01 \mathrm{M}$ citrate buffer ( $\mathrm{pH} \mathrm{6.0)}$ ) for $2 \mathrm{~min} . \mathrm{H}_{2} \mathrm{O}_{2}$ was employed to inhibit endogenous peroxide and non-immune goat serum (Fuzhou Maixin Biotech Co., Ltd.) was used to reduce non-specific antibody binding at room temperature for $15 \mathrm{~min}$. Sections were then incubated with LATS1 antibody (1:200; cat. no. 9153; Cell Signaling Technology, Inc., Danvers, MA, USA) at $4^{\circ} \mathrm{C}$ overnight. Biotinylated anti-rabbit horseradish peroxidase polymer (ready to use; cat. no. 9922; Fuzhou Maixin Biotech Co., Ltd.) was used as a secondary antibody at $37^{\circ} \mathrm{C}$ for $2 \mathrm{~h}$. Following washing, the peroxidase reaction was developed with 3,3-diaminobenzidine. Counterstaining with hematoxylin was performed and the sections were dehydrated in ethanol prior to mounting.

Two independent blinded investigators examined all tumor slides randomly. As in previous studies $(13,14)$, immunostaining of LATS1 was scored on a semi-quantitative scale by evaluating the intensity and percentage of tumor cells stained. Cytoplasmic immunostaining was regarded as positive. The intensity of LATS1 staining was scored as follows: 0 , weak/negative; 1, moderate; or 2 (strong). Staining percentage, the percentage of tumor cells stained, was scored as follows: $1,1-25 \% ; 2,26-50 \% ; 3,51-75 \%$; or $4,76-100 \%$. Total score was obtained by multiplying the staining and percentage scores. Specimens with a total score of 5-8 were considered to be positive for LATS1 expression. Specimens with a total score of 0-4 were considered to have low LATS1 expression.

Cell culture and transfection. Caski, HeLa and SiHa cell lines were obtained from the American Type Culture Collection (Manassas, VA, USA). SiHa and HeLa cells were cultured in minimum essential medium (MEM; Invitrogen; Thermo Fisher Scientific, Inc., Waltham, MA, USA) and Caski cells were cultured in RPMI-1640 medium containing $10 \%$ fetal bovine serum (Gibco; Thermo Fisher Scientific, Inc.) at $37^{\circ} \mathrm{C}$ for 2 days. Cells were cultured on sterilized culture plates and were passaged every two days with trypsin.

The plasmid of pCMV6-LATS1 was purchased from (OriGene Technologies, Inc. (Rockville, MD, USA) Plasmid was transfected into cells using Lipofectamine ${ }^{\circledR} 3000$ reagent (Invitrogen; Thermo Fisher Scientific, Inc.) at $37^{\circ} \mathrm{C}$ for $48 \mathrm{~h}$. pCMV6 empty vector was used as a negative control. For transient knockdown experiments, oligonucleotide pools of small interfering RNA (siRNA) targeting LATS1 and non-targeting siRNA (cat. no. M-004632-00-0005) were purchased from Dharmacon (Lafayette, CO, USA) and transfected using Lipofectamine ${ }^{\circledR} 2000$ (Invitrogen; Thermo Fisher Scientific, Inc.) at $37^{\circ} \mathrm{C}$ for $48 \mathrm{~h}$, according to the manufacturersprotocols.

Western blot analysis. Proteins were extracted and quantified using the Bradford method, and $20 \mu \mathrm{g}$ protein was separated by SDS-PAGE (5\% stacking gel and 10\% separating gel). Proteins were transferred to polyvinylidene fluoride membranes and $5 \%$ BSA solution (w/v) was used to reduce non-specific antibody binding at room temperature for $1 \mathrm{~h}$. Proteins were incubated overnight at $4^{\circ} \mathrm{C}$ with antibodies against LATS1 (1:1,000; cat. no. 9153; Cell Signaling Technology, Inc.), phosphorylated-yes associated protein 1 (p-YAP; 1:1,000; cat. no. 4911; Cell Signaling Technology, Inc.), YAP (1:1,000; cat. no. 4912; Cell Signaling Technology, Inc.), p27 (1:1,000; cat. no. 2552; Cell Signaling Technology, Inc.), cyclin E (1:1,000; cat. no. 20808; Cell Signaling Technology, Inc.), connective tissue growth factor (CTGF; 1:1,000; 23936-1-AP; Proteintech, Chicago, Illinois, USA) and matrix metalloproteinase 9 (MMP9; 1:1,000; cat. no. 3852; Cell Signaling Technology, Danvers, MA, USA) and GAPDH (1:1,000; cat. no. sc-25778; Santa Cruz Biotechnology, Inc., Dallas, TX, USA). PVDF membranes were washed using TBST solution (Sigma-Aldrich; Merck Millipore, Darmstadt, Germany) three times for $5 \mathrm{~min}$ each. Following incubation with peroxidase-coupled anti-mouse/rabbit IgG (1:2,000; cat. no. 5127/58802; Cell Signaling Technology, Inc., Danvers, MA, USA) at $37^{\circ} \mathrm{C}$ for $2 \mathrm{~h}$, proteins were visualized using SuperSignal West Dura Extended Duration Substrate (Thermo Fisher Scientific, Inc.) and detected using a DNR Bio-Imaging System (DNR Bio-Imaging Systems, Ltd., Jerusalem, Israel).

MTT assay. Cells were plated in 96-well plates in MEM containing $10 \%$ fetal bovine serum at approximately $1,000-1500$ cells per well. For the measurement of cell viability, $20 \mu \mathrm{l}$ MTT solution was added to each well and incubated at $37^{\circ} \mathrm{C}$ for $4 \mathrm{~h}$. Subsequently, the remaining MTT formazan was dissolved in $150 \mu \mathrm{l}$ dimethyl sulfoxide. The plate was measured at a wavelength of $490 \mathrm{~nm}$ using a plate reader.

Cell invasion analysis. Cell invasion was examined using Transwell assay with 24-well Transwell chambers. Briefly, Transwell chamber inserts were coated using 20-25 $\mu$ l Matrigel from BD Biosciences (Franklin Lakes, NJ, USA) with a dilution of 1:5. $\sim 48 \mathrm{~h}$ after cell transfection, $\sim 1 \times 10^{5}$ cells were re-suspended in $100 \mu \mathrm{l}$ of serum-free MEM and were added to the upper chamber. MEM with $10-15 \%$ fetal bovine serum was added to the lower chamber. After incubation at $37^{\circ} \mathrm{C}$ for 16-20 h, cells on the upper side of membranes were removed using a cotton swab and the cells that had invaded through the filter were washed with phosphate-buffered saline and visualized with hematoxylin at room temperature for $5 \mathrm{~min}$. The number of invaded cells was counted in 5 randomly selected high power fields using a light microscope. This experiment was performed in triplicate.

Statistical analysis. SPSS version 11.5 for Windows (SPSS, Inc., Chicago, IL, USA) was used for all statistical analyses. Data was presented as mean \pm standard deviation. $\chi^{2}$ test was used to examine potential associations between LATS1 expression and clinicopathological factors and a paired Student-s $t$-test was used to compare other data generated from LATS1-transfected or knockdown cells. $\mathrm{P}<0.05$ was considered to indicate a statistically significant difference.

\section{Results}

Clinical significance of LATS1 in human cervical cancer. LATS1 expression was located in the cytoplasm in normal cervical tissues (Fig. 1A). Of the 80 cervical cancer tissues, 46 of them (45\%) exhibited decreased LATS1 staining, although others were positive for LATS1 expression (Fig. 1B-D). 
A

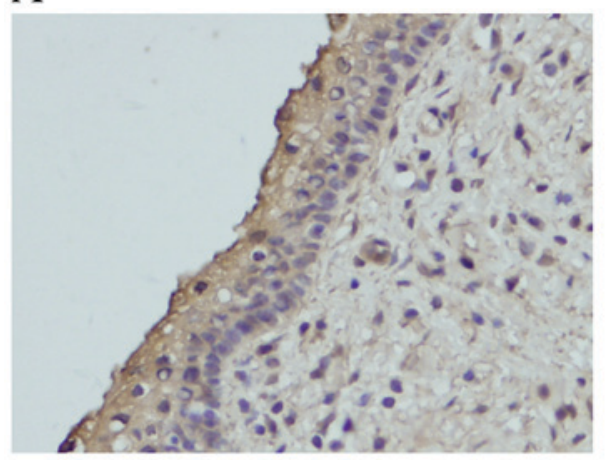

$\mathrm{C}$

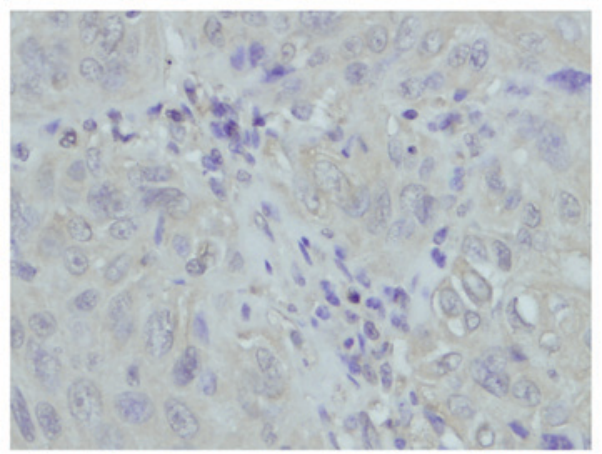

B

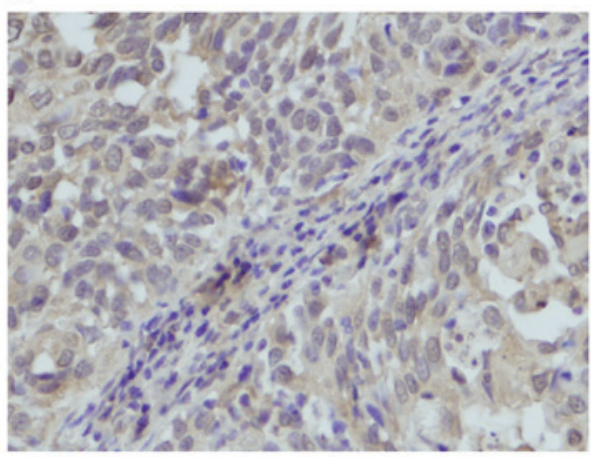

$\mathrm{D}$

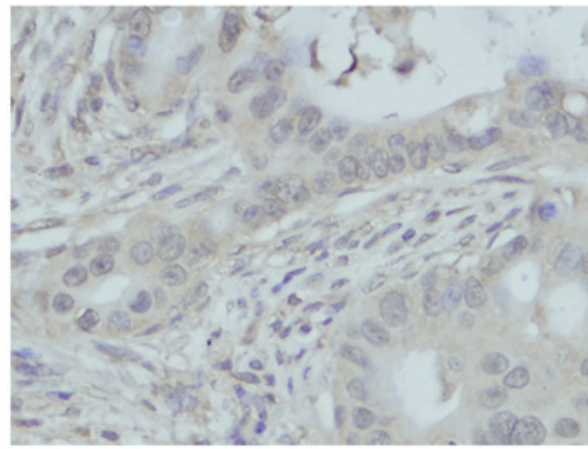

Figure 1. Expression of LATS1 protein in cervical cancer tissues. (A) Strong LATS1 expression in normal cervical tissue. (B) Positive LATS1 expression in a case of stage II cervical cancer. (C) Negative staining of LATS1 in a case of stage III cervical cancer. (D) Weak LATS1 expression in a case of stage I adenocarcinoma. Magnification, x400. LATS1, large tumor suppressor kinase 1 .

The potential association of low LATS1 expression with clinicopathological characteristics was analyzed. The results demonstrated that low LATS1 immunostaining in cervical cancer was significantly associated with primary tumor, node, metastasis (TNM) stage (stages II+III vs. stage I, $\mathrm{P}=0.0102$ ) and primary tumor $(\mathrm{T})$ stage $(\mathrm{TII}+\mathrm{III}$ vs. TI, $\mathrm{P}=0.0217)$. No significant association was identified between low LATS1 expression and other parameters, including age, histological type and differentiation (Table I).

LATS1 suppresses proliferation and invasion of cervical cancer cells. LATS1 was examined by western blot analysis in cervical cancer cell lines (Fig. 2A). The present study demonstrated that SiHa cells had low LATS1 protein expression and Caski cells had relatively high LATS1 protein expression. To determine its biological roles in cervical cancer cell lines, plasmid transfection was performed in SiHa cells and siRNA knockdown was performed in Caski cells. As presented in Fig. 2B, LATS1 transfection increased protein levels of LATS1 in $\mathrm{SiHa}$ cells and siRNA reduced LATS1 protein levels in Caski cells. The MTT assay demonstrated that LATS1 upregulation significantly decreased cell growth rate compared with cells treated with empty plasmid. (SiHa:P=0.019; Caski:P=0.006, Fig. 3A). A Matrigel invasion assay was also performed to assess the effect of LATS1 on cell invasion. As presented in Fig. 3B, LATS1 transfection significantly decreased the invasiveness of SiHa cells compared with cells transfected with empty plasmid $(\mathrm{P}=0.007)$, while LATS1 knockdown significantly increased the invasiveness of Caski cells compared with cells treated with negative siRNA $(\mathrm{P}=0.005)$.

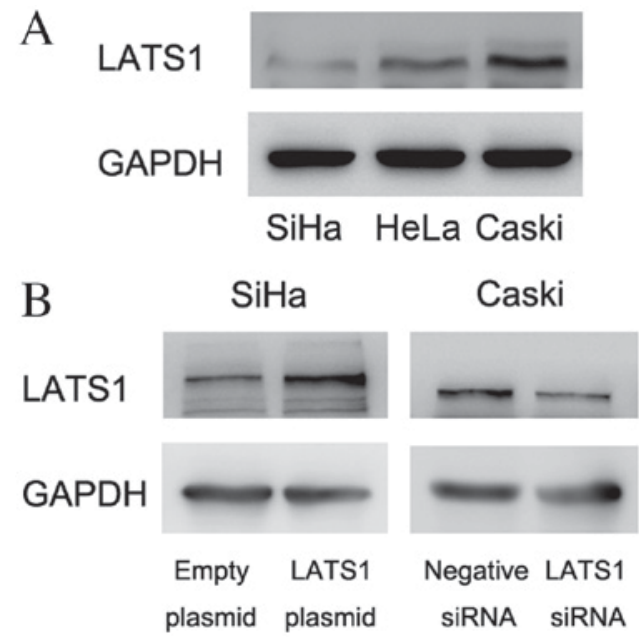

Figure 2. LATS1 transfection efficiency in cervical cancer cell lines. (A) Western blot analysis demonstrated strong LATS1 expression in the Caski cell line and weak LATS1 expression in the SiHa cell line. (B) Western blot analysis demonstrated that LATS1 transfection in SiHa cells upregulated LATS1 protein expression, and siRNA knockdown in Caski cells downregulated LATS1 protein expression, compared with controls. LATS1, large tumor suppressor kinase 1; siRNA, small interfering RNA.

LATS1 regulates the expression of cyclin E, p27, MMP9 and $Y A P$ in cervical cancer cells. In order to investigate the molecular mechanism underlying LATS1-induced cell growth and invasion, the present study examined the expression of growth and invasion-associated proteins. The results demonstrated that the cyclin E expression was notably decreased following LATS1 
Table I. Distribution of LATS1 status in cervical carcinoma according to clinicopathological characteristics.

\begin{tabular}{|c|c|c|c|c|}
\hline Characteristic & $\begin{array}{l}\text { Total } \\
\text { number of } \\
\text { patients }\end{array}$ & $\begin{array}{c}\text { Number of } \\
\text { patients with low } \\
\text { LATS1 expression }\end{array}$ & $\begin{array}{l}\text { Number of } \\
\text { patients with positive } \\
\text { LATS1 expression }\end{array}$ & P-value \\
\hline Age & & & & 0.5445 \\
\hline$<50$ & 55 & 29 & 26 & \\
\hline$\geq 50$ & 25 & 15 & 10 & \\
\hline Histological type & & & & 0.4552 \\
\hline Squamous cell carcinoma & 71 & 38 & 33 & \\
\hline Adenocarcinoma & 9 & 6 & 3 & \\
\hline Differentiation & & & & 0.2905 \\
\hline Well/moderate & 58 & 34 & 24 & \\
\hline Poor & 22 & 10 & 12 & \\
\hline TNM stage & & & & 0.0102 \\
\hline I & 32 & 12 & 20 & \\
\hline II+III & 48 & 32 & 16 & \\
\hline T stage & & & & 0.0217 \\
\hline $\mathrm{T} 1$ & 42 & 18 & 24 & \\
\hline $\mathrm{T} 2+3$ & 38 & 26 & 12 & \\
\hline Lymph node metastasis & & & & 0.2458 \\
\hline Negative & 50 & 25 & 25 & \\
\hline Positive & 30 & 19 & 11 & \\
\hline
\end{tabular}

The P-value compares the patients with high expression of LATS1 in different groups. LATS1, large tumor suppressor kinase 1; TNM, primary tumor, node, metastasis; T stage, primary tumor stage.

overexpression in SiHa cells, compared with cells treated with empty plasmid, and increased following LATS1 knockdown in Caski cells, compared with cells treated with negative siRNA. Protein expression of cell cycle inhibitor p27 was increased compared to control following LATS1 transfection in SiHa cells and decreased compared to the control following LATS1 knockdown in Caski cells. (Fig. 4A). The present study also examined invasion-associated protein MMP9, and demonstrated that MMP9 was downregulated in LATS1-transfected SiHa cells and upregulated in LATS1-knockdown Caski cells, compared to respective controls (Fig. 4A). LATS1 is the upstream inhibitor of Hippo signaling. Activation of the Hippo signaling pathway inhibits the expression and function of YAP oncoprotein and suppresses cancer growth and invasion. The current study examined YAP expression, and its downstream factor CTGF, in LATS1 overexpressing and knockdown cervical cancer cells. As presented in Fig. 4B, in LATS1-transfected SiHa cells, CTGF and YAP levels were decreased, compared to the control SiHa cells. In LATS1 knockdown Caski cells, their expression (YAP and CTGF) was upregulated compared with control Caski cells. Changes in the levels of YAP phosphorylation were also examined. Western blot analysis demonstrated that LATS1 increased the phosphorylation of YAP.

\section{Discussion}

LATS1 is the mammalian homolog of Drosophila LATS, originally identified as a cell proliferation inhibitor (15). It is a serine/threonine kinase that localizes to the mitotic apparatus. LATS1 may form a complex with cyclin-dependent kinase 1 in early mitosis, decreasing $\mathrm{H} 1$ histone kinase activity, which indicates its role as a cell cycle inhibitor (16). LATS1-knockout mice developed sarcomas and ovarian tumors, indicating that it may also function as a tumor suppressor (3). The expression and biological roles of LATS1 have been implicated in a number of human malignancies, including gastric cancer, breast cancer, glioma, renal cell carcinoma and ovarian cancer $(9,11,17-19)$. However, to the best of our knowledge, the involvement of LATS1 in human cervical carcinoma has not been reported. The present study examined LATS1 protein expression in 80 cases of cervical cancer and demonstrated that LATS1 was downregulated in $45 \%$ of tissue specimens. Statistical analysis demonstrated that LATS1 downregulation was positively associated with advanced TNM stage and tumor status, indicating that loss of LATS1 is associated with cervical cancer progression. The results of the current study are consistent with previous studies (20-22), indicating that LATS1 may be a potential tumor suppressor in human cervical cancer.

To identify its biological roles in cervical cancer cells, the present study transfected SiHa cells with a LATS1 plasmid and Caski cells were transfected with LATS1 siRNA. Subsequently, the effects on cell proliferation and invasion were examined. Consistent with the immunohistochemical results of the present study, LATS1 transfection significantly inhibited cell growth and invasiveness of SiHa cells, while LATS1 siRNA had the opposite effect in Caski cells. The current study further investigated 

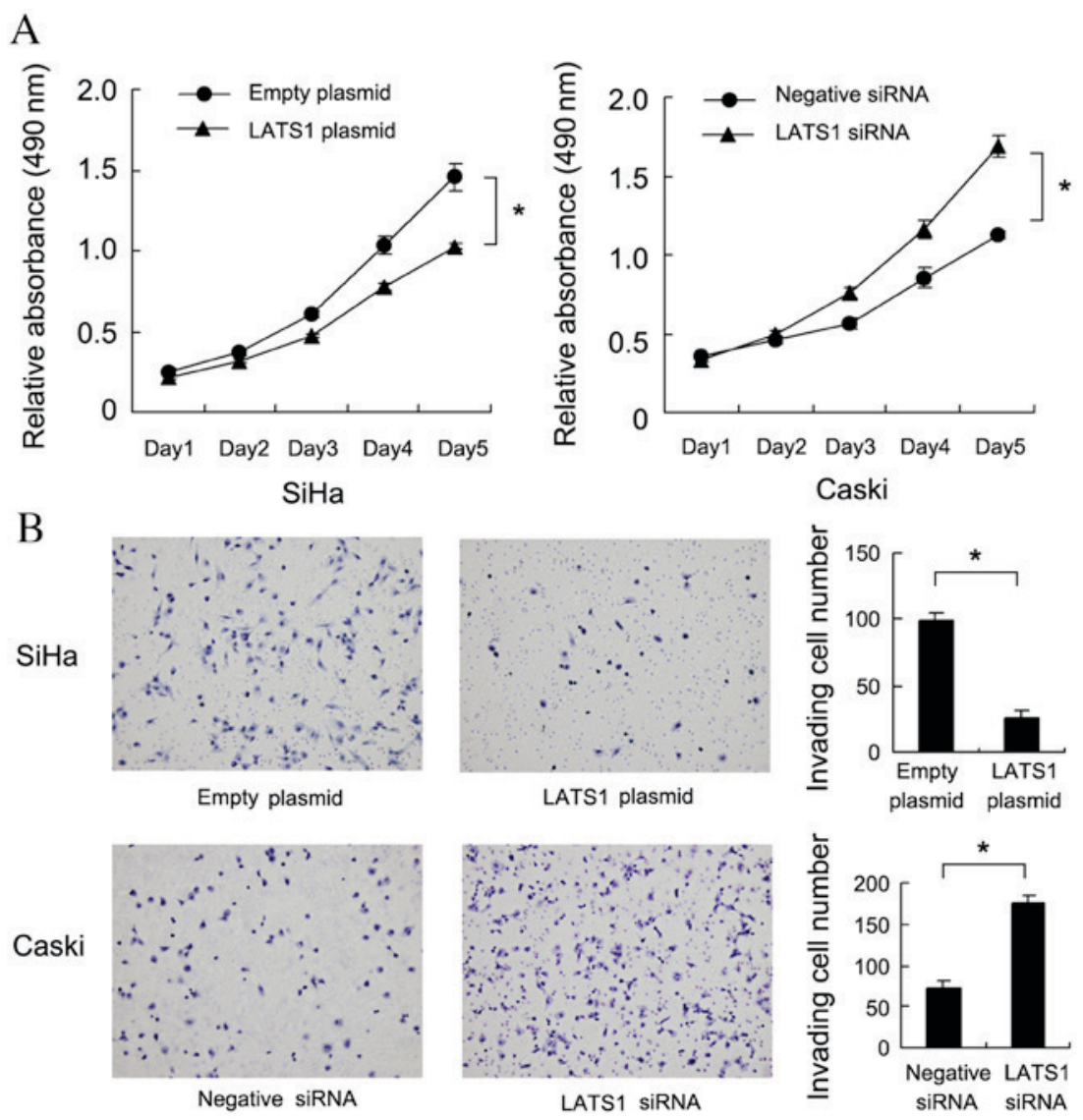

Figure 3. LATS1 inhibits proliferation and invasion of cervical cancer cells. (A) MTT assay demonstrated that LATS1 transfection significantly reduced cell proliferation in the SiHa cell line ("P<0.05 vs. empty plasmid control), while LATS1 knockdown significantly increased cell proliferation in the Caski cell line ("P<0.05 vs. negative siRNA control). (B) Matrigel invasion assay demonstrated that LATS1 transfection in SiHa cells significantly decreased the number of invading cells (" $\mathrm{P}<0.05$ vs. empty plasmid control), while LATS1 knockdown in Caski cells significantly increased the invasiveness of Caski cells (" $\mathrm{P}<0.05$ vs. negative siRNA control). LATS1, large tumor suppressor kinase 1; siRNA, small interfering RNA.

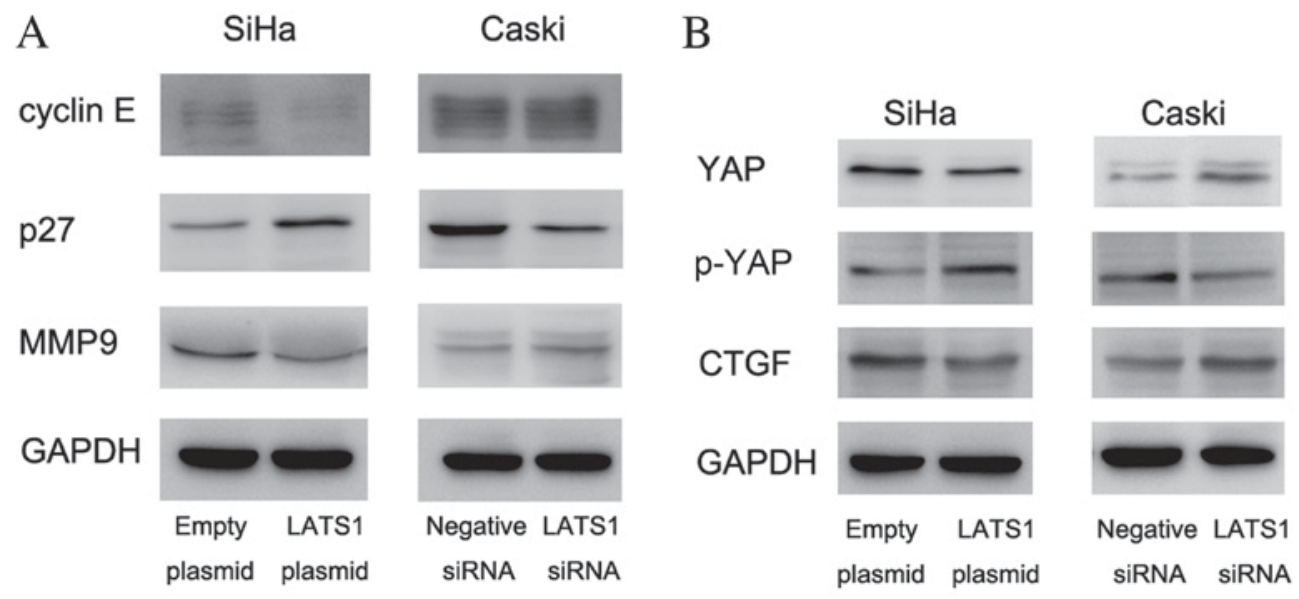

Figure 4. LATS1 inhibits cervical cancer cell proliferation and invasion via regulation of cyclin E, p27, MMP9, YAP and CTGF. (A) Western blot analysis demonstrated that LATS1 transfection downregulated the levels of cyclin E and MMP9, and upregulated p27 expression in SiHa cells compared with the control. LATS1 knockdown in Caski cells upregulated cyclin E and MMP9, and downregulated p27 expression. (B) LATS1 overexpression in SiHa cells decreased YAP and CTGF protein levels, and increased YAP phosphorylation compared with the control. LATS1 knockdown in Caski cells resulted in the opposite effects, which upregulated YAP and downregulated p-YAP. LATS1, large tumor suppressor kinase 1; MMP9, matrix metalloproteinase 9; YAP, yes associated protein 1; p-YAP, phosphorylated YAP; CTGF, connective tissue growth factor; siRNA, small interfering RNA.

the potential underlying mechanisms by which LATS1 inhibited cell proliferation and invasion in cervical cancer. Previous studies demonstrated that LATS1 functions as a cell cycle regulator $(20,21)$. Thus, the protein levels of cyclin E and p27 in LATS1-overexpressing SiHa cells and LATS1-knockdown Caski cells were investigated in the present study. The results demonstrated that LATS1 downregulated cyclin E and upregulated p27. Cyclin E facilitates and p27 inhibits cell cycle 
progression $(23,24)$. Cycle E overexpression is important during cervical cancer proliferation (25), and p27 downregulation has been previously identified in cervical cancer tissues where it may cause cell cycle arrest in cervical cancer cells $(26,27)$. In addition, the present study demonstrated that invasion-associated protein MMP9 was negatively regulated by LATS1 expression. MMP9 is considered to be associated with cervical cancer invasion (28-30). Thus LATS1 may regulate cervical cancer growth and invasion through modulation of cell cycle proteins and MMP9. As LATS1 is the upstream positive regulator of Hippo pathway, the current study investigated changes in the expression levels of downstream proteins, including YAP and CTGF. The results demonstrated that LATS1 overexpression inhibited CTGF and YAP expression, and increased YAP phosphorylation in SiHa cells compared with control SiHa cells. YAP is a positive regulator of growth and invasion in various types of human cancers, including cervical cancer (31). CTGF is the target of YAP and functions as a growth promoter. CTGF has been reported to positively regulate the cell cycle and MMP proteins $(32,33)$. Thus, the role of LATS1 in cervical cancer invasion and proliferation may depend on its regulation on YAP, and its downstream target CTGF.

In conclusion, LATS1 is downregulated in human cervical cancers and LATS1 expression is associated with TNM stage. LATS1 inhibited cervical cancer cell proliferation and invasion, potentially through regulation of $\mathrm{p} 27$ and MMP9. LATS1 may activate the Hippo pathway through downregulation of YAP and CTGF. Loss of LATS1 may serve as an indicator of malignant phenotype in human cervical cancer.

\section{Acknowledgements}

The present study was supported by the Yunnan Provincial Science and Technology Project Fund (grant no. 2014FB200).

\section{References}

1. Siegel R, Ma J, Zou Z and Jemal A: Cancer statistics, 2014. CA Cancer J Clin 64: 9-29, 2014.

2. Song C, Zhu S, Wu C and Kang J: Histone deacetylase (HDAC) 10 suppresses cervical cancer metastasis through inhibition of matrix metalloproteinase (MMP) 2 and 9 expression. J Biol Chem 288: 28021-28033, 2013.

3. St John MA, Tao W, Fei X, Fukumoto R, Carcangiu ML, Brownstein DG, Parlow AF, McGrath J and Xu T: Mice deficient of Lats1 develop soft-tissue sarcomas, ovarian tumours and pituitary dysfunction. Nat Genet 21: 182-186, 1999.

4. Huntoon CJ, Nye MD, Geng L, Peterson KL, Flatten KS, Haluska P, Kaufmann SH and Karnitz LM: Heat shock protein 90 inhibition depletes LATS1 and LATS2, two regulators of the mammalian hippo tumor suppressor pathway. Cancer Res 70 8642-8650, 2010.

5. Furth N, Bossel Ben-Moshe N, Pozniak Y, Porat Z, Geiger T, Domany E, Aylon Y and Oren M: Down-regulation of LATS kinases alters p53 to promote cell migration. Genes Dev 29: 2325-2330, 2015.

6. Visser S and Yang X: LATS tumor suppressor: A new governor of cellular homeostasis. Cell Cycle 9: 3892-3903, 2010.

7. Morinaga N, Shitara Y, Yanagita Y, Koida T, Kimura M, Asao T, Kimijima I, Takenoshita S, Hirota T, Saya H and Kuwano H: Molecular analysis of the h-warts/LATS1 gene in human breast cancer. Int J Oncol 17: 1125-1129, 2000.

8. Wierzbicki PM, Adrych K, Kartanowicz D, Stanislawowski M, Kowalczyk A, Godlewski J, Skwierz-Bogdanska I, Celinski K, Gach T, Kulig J, Korybalski B and Kmiec Z: Underexpression of LATS1 TSG in colorectal cancer is associated with promoter hypermethylation. World J Gastroenterol 19: 4363-4373, 2013.
9. Xu ZP, Zhu JS, Zhang Q and Wang XY: A breakdown of the Hippo pathway in gastric cancer. Hepatogastroenterology 58: 1611-1617, 2011.

10. Lin XY, Zhang XP, Wu JH, Qiu XS and Wang EH: Expression of LATS1 contributes to good prognosis and can negatively regulate YAP oncoprotein in non-small-cell lung cancer. Tumour Biol 35: 6435-6443, 2014.

11. Xu B, Sun D, Wang Z, Weng H, Wu D, Zhang X, Zhou Y and $\mathrm{Hu}$ W: Expression of LATS family proteins in ovarian tumors and its significance. Hum Pathol 46: 858-867, 2015.

12. Puppo P, Conti G, Francesca F, Mandressi A and Naselli A; AURO. it guideline committee: New Italian guidelines on bladder cancer, based on the World Health Organization 2004 classification. BJU Int 106: 168-179, 2010.

13. Bocchi EA, Tanigawa RY, Brandão SM, Cruz F, Issa V, Ayub-Ferreira S, Chizzola P, Souza G, Fiorelli AI, Bacal F, et al: Immunohistochemical quantification of inflammatory cells in endomyocardial biopsy fragments after heart transplantation: A new potential method to improve the diagnosis of rejection after heart transplantation. Transplant Proc 46: 1489-1496, 2014.

14. Roussel AJ, Knol AC, Bourdeau PJ and Bruet V: Optimization of an immunohistochemical method to assess distribution of tight junction proteins in canine epidermis and adnexae. J Comp Pathol 150: 35-46, 2014

15. Justice RW, Zilian O, Woods DF, Noll M and Bryant PJ: The Drosophila tumor suppressor gene warts encodes a homolog of human myotonic dystrophy kinase and is required for the control of cell shape and proliferation. Genes Dev 9: 534-546, 1995.

16. Tao W, Zhang S, Turenchalk GS, Stewart RA, St John MA, Chen W and Xu T: Human homologue of the Drosophila melanogaster lats tumour suppressor modulates CDC2 activity. Nat Genet 21: 177-181, 1999.

17. Ji T, Liu D, Shao W, Yang W, Wu H and Bian X: Decreased expression of LATS1 is correlated with the progression and prognosis of glioma. J Exp Clin Cancer Res 31: 67, 2012.

18. Takahashi Y, Miyoshi Y, Takahata C, Irahara N, Taguchi T, Tamaki Y and Noguchi S: Down-regulation of LATS1 and LATS2 mRNA expression by promoter hypermethylation and its association with biologically aggressive phenotype in human breast cancers. Clin Cancer Res 11: 1380-1385, 2005.

19. Chen KH, He J, Wang DL, Cao JJ, Li MC, Zhao XM, Sheng X, Li WB and Liu WJ: Methylation-associated inactivation of LATS1 and its effect on demethylation or overexpression on YAP and cell biological function in human renal cell carcinoma. Int J Oncol 45: 2511-2521, 2014.

20. Visser-Grieve S, Zhou Z, She YM, Huang H, Cyr TD, Xu T and Yang X: LATS1 tumor suppressor is a novel actin-binding protein and negative regulator of actin polymerization. Cell Res 21: 1513-1516, 2011.

21. Hao Y, Chun A, Cheung K, Rashidi B and Yang X: Tumor suppressor LATS1 is a negative regulator of oncogene YAP. J Biol Chem 283: 5496-5509, 2008.

22. Xia H, Qi H, Li Y, Pei J, Barton J, Blackstad M, Xu T and Tao W: LATS1 tumor suppressor regulates G2/M transition and apoptosis. Oncogene 21: 1233-1241, 2002.

23. Lopez-Beltran A, MacLennan GT and Montironi R: Cyclin E as molecular marker in the management of breast cancer: A review. Anal Quant Cytol Histol 28: 111-114, 2006.

24. Guan X, Wang Y, Xie R, Chen L, Bai J, Lu J and Kuo MT: p27 (Kip1) as a prognostic factor in breast cancer: A systematic review and meta-analysis. J Cell Mol Med 14: 944-953, 2010.

25. Zubillaga-Guerrero MI, Alarcón-Romero Ldel C, Illades-Aguiar B, Flores-Alfaro E, Bermúdez-Morales VH, Deas J and Peralta-Zaragoza O: MicroRNA miR-16-1 regulates CCNE1 (cyclin E1) gene expression in human cervical cancer cells. Int J Clin Exp Med 8: 15999-16006, 2015.

26. Prasad SB, Yadav SS, Das M, Modi A, Kumari S, Pandey LK, Singh S, Pradhan S and Narayan G: PI3K/AKT pathway-mediated regulation of $\mathrm{p} 27$ (Kip1) is associated with cell cycle arrest and apoptosis in cervical cancer. Cell Oncol (Dordr) 38: 215-225, 2015.

27. van de Putte G, Holm R, Lie AK, Tropé CG and Kristensen GB: Expression of p27, p21, and p16 protein in early squamous cervical cancer and its relation to prognosis. Gynecol Oncol 89: 140-147, 2003.

28. Zhu D, Ye M and Zhang W: E6/E7oncoproteins of high risk HPV-16 upregulate MT1-MMP, MMP-2 and MMP-9 and promote the migration of cervical cancer cells. Int J Clin Exp Pathol 8: 4981-4999, 2015. 
29. Pahne-Zeppenfeld J, Schröer N, Walch-Rückheim B, Oldak M, Gorter A, Hegde S and Smola S: Cervical cancer cell-derived interleukin-6 impairs CCR7-dependent migration of MMP-9-expressing dendritic cells. Int J Cancer 134: 2061-2073, 2014.

30. Roomi MW, Monterrey JC, Kalinovsky T, Rath M and Niedzwiecki A: In vitro modulation of MMP-2 and MMP-9 in human cervical and ovarian cancer cell lines by cytokines, inducers and inhibitors. Oncol Rep 23: 605-614, 2010.

31. He C, Mao D, Hua G, Lv X, Chen X, Angeletti PC, Dong J, Remmenga SW, Rodabaugh KJ, Zhou J, et al: The Hippo/YAP pathway interacts with EGFR signaling and HPV oncoproteins to regulate cervical cancer progression. EMBO Mol Med 7: $1426-1449,2015$
32. Zhen Y, Ye Y, Yu X, Mai C, Zhou Y, Chen Y, Yang H, Lyu X, Song Y, Wu Q, et al: Reduced CTGF expression promotes cell growth, migration, and invasion in nasopharyngeal carcinoma. PLoS One 8: e64976, 2013.

33. Tsai HC, Su HL, Huang CY, Fong YC, Hsu CJ and Tang CH CTGF increases matrix metalloproteinases expression and subsequently promotes tumor metastasis in human osteosarcoma through down-regulating miR-519d. Oncotarget 5: 3800-3812, 2014. 